\title{
Which is the Dominant Nonlinearity in Long-haul PDM-QPSK Coherent Transmissions?
}

\author{
A. Bononi, P. Serena, N. Rossi, D. Sperti
}

Università degli Studi di Parma, Dipartimento di Ingegneria dell'Informazione, v.le G. Usberti 181/A, 43124 Parma (Italy), 凶alberto.bononi@unipr.it

\begin{abstract}
The contribution of each nonlinear effect to system penalty is investigated in a $20 \times 100 \mathrm{~km}$ homogeneous WDM PDM-QPSK link over wide range of symbol rates and setups. XPolM is found to dominate $112 \mathrm{~Gb} / \mathrm{s}$ dispersion-managed links.
\end{abstract}

\section{Introduction}

The recent development of digital signal processing (DSP) based coherent detection in optical networks expedited the use of polarization division multiplexing (PDM) as a cost-effective way of doubling system capacity. With wavelength division multiplexing (WDM), the cross-nonlinearities make neighboring channels interact depending on their power and state of polarization (SOP). The last is of particular concern in PDM systems since they are more sensitive to a new kind of distortion that has been generally referred to as cross-polarization modulation (XPolM) ${ }^{1}$ as a way to distinguish it from the well known crossphase modulation (XPM). Experiments showed that XPoIM depends on a larger number of channels than $\mathrm{XPM}^{2}$, while Winter et al. ${ }^{3}$ provided a model that successfully measured the degree of polarization degradation in presence of XPoIM. Despite these results, it is still not clear when the bit error rate (BER) is dominated by XPoIM and how XPolM relates to the other relevant nonlinear effects, such as XPM and self-phase modulation (SPM).

Aim of this paper is to fill the gap, by providing a systematic simulation study of system performance where each nonlinear effect acts individually. Our goal is to determine for the PDMquadrature phase shift keying (QPSK) modulation format which is the dominant nonlinearity over both dispersion-managed (DM) and dispersion un-managed (noDM) systems, thus extending the results of ${ }^{4,5}$.

\section{Separation of Nonlinear Effects}

The propagation of an optical field $\vec{A}(z, \tau)$ within a nonlinear randomly birefringent optical fiber with negligible polarization mode dispersion (PMD) can be numerically evaluated through the splitstep Fourier algorithm (SSFA) applied to the Manakov equation ${ }^{6}$. We show here how to separate nonlinear effects in the nonlinear step of the SSFA in WDM propagation. Let the WDM signal be $\vec{A}(z, \tau)=\sum_{k} \overrightarrow{A_{k}}(z, \tau) \exp ^{i 2 \pi k \Delta f \tau}$, with channel spacing $\Delta f$. The Manakov nonlinear step in absence of four wave mixing (FWM) is 3,6

$$
\frac{\partial \vec{A}_{n}}{\partial z}=-i \gamma\left(\left(A_{n}^{2}+\frac{3}{2} \sum_{k \neq n} A_{k}^{2}\right) \sigma_{0}+\frac{1}{2} \sum_{k \neq n}\left(\vec{a}_{k} \cdot \vec{\sigma}\right)\right) \vec{A}_{n}
$$

where: $\gamma$ is the nonlinear index; $A_{k}^{2}$ are the signal intensities; $\sigma_{0}$ is the $2 \times 2$ identity matrix; $\vec{\sigma}$ is the Pauli vector; and $\vec{a}_{k}$ is the real 3D Stokes vector associated with the Jones vector $\vec{A}_{k}$. Equation (1) indicates that in birefringent media the Kerr effect may change the SOP of the signals (i.e. induce XPoIM) only through the scalar product $\vec{a}_{k} \cdot \vec{\sigma}$, while the other terms depend on the signal intensities only, like in scalar propagation. Therefore, following $^{3}$, we call SPM the term proportional to $A_{n}^{2}$, XPM the one proportional to $\frac{3}{2} \sum_{k \neq n} A_{k}^{2}$, and all the rest XPoIM.

At each time instant, the exact solution of the nonlinear step (1) is

$$
\vec{A}_{n}(z)=e^{\frac{-j \gamma L\left(A_{n}^{2}+3 \sum_{k \neq n} A_{k}^{2}\right)}{2}} \underline{e}^{\frac{-j \gamma L}{2}\left(\vec{s}_{t} \cdot \vec{\sigma}\right)} \vec{A}_{n}(0)
$$

where $\vec{s}_{t}=\sum_{k} \vec{a}_{k}(0)$ is the real pivot (the summation extends to all channels), $L$ is the step effective length, and $\underline{e}^{-j(\vec{v} \cdot \vec{\sigma})}=\cos (|\vec{v}|) \sigma_{0}-$ $j \sin (|\vec{v}|)\left(\frac{\vec{v}}{|\vec{v}|} \cdot \vec{\sigma}\right)$ is a matrix exponential for real vector $\vec{v}$. Equation (2) accounts for all effects without distinguishing among them. The solution of (1) for each individual effect gives instead:

$$
\begin{aligned}
\vec{A}_{\mathrm{spm}}(z) & =e^{-j \gamma L A_{n}^{2} \vec{A}(0)} \\
\vec{A}_{\mathrm{xpm}}(z) & =e^{-j \gamma L \frac{3}{2}} \sum_{k \neq n} A_{k}^{2} \vec{A}(0) \\
\vec{A}_{\text {XpolM }}(z) & =e^{\frac{j \gamma L A_{n}^{2}}{2}} \underline{e}^{\frac{-j \gamma L}{2}\left(\vec{s}_{t} \cdot \vec{\sigma}\right)} \vec{A}(0) .
\end{aligned}
$$

In the next sections we quantify the impact of each nonlinear effect. 

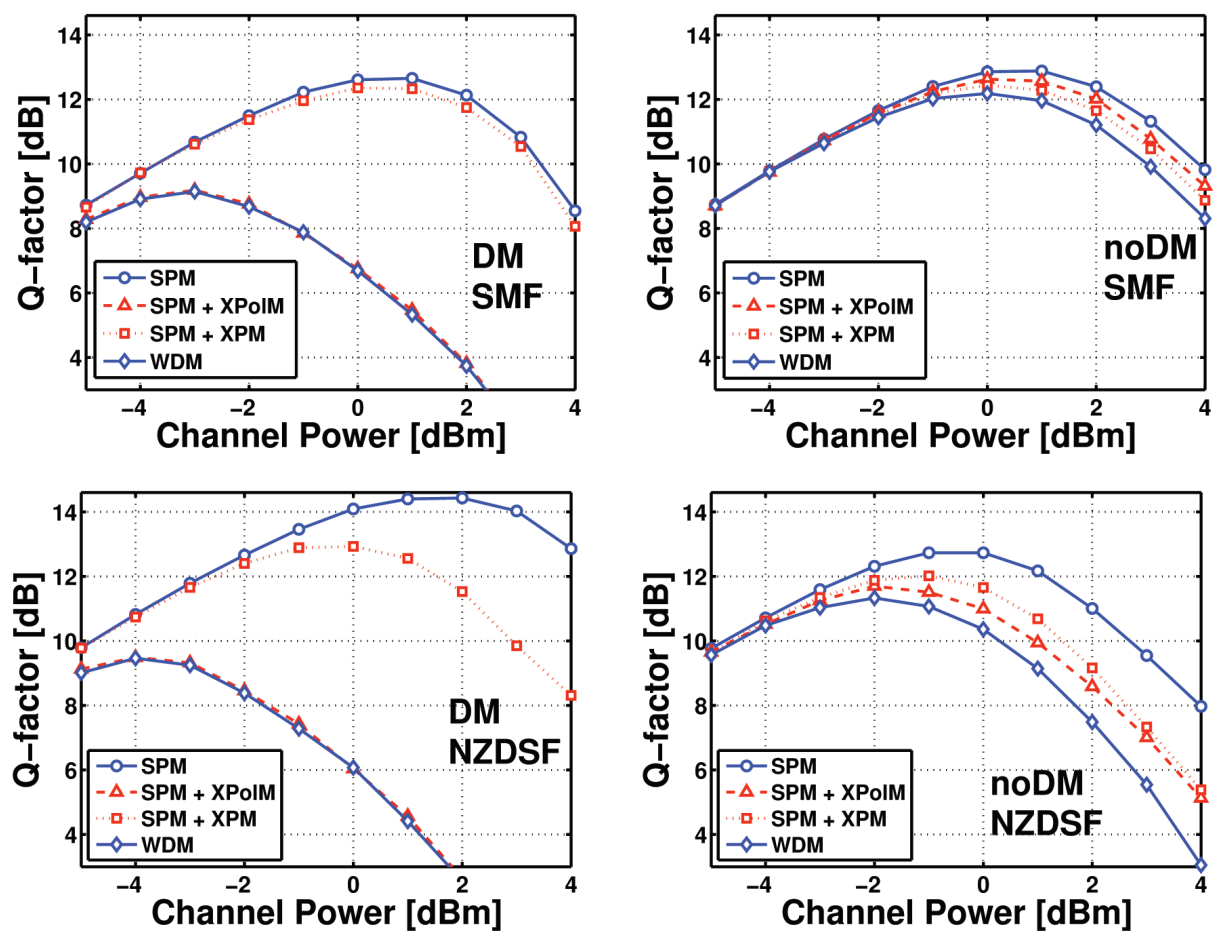

Fig. 1: Avg. Q-factor vs. power for homogeneous 19-channel 112 Gbit/s PDM-QPSK 20x100km link, 50 GHz grid.

\section{Numerical setup}

We simulated both a dispersion managed and un-managed $20 \times 100 \mathrm{~km}$ link with the opensource software Optilux ${ }^{7}$. In both cases the transmission fiber had nonlinear index $\gamma=1.3$ $\mathrm{W}^{-1} \mathrm{~km}^{-1}$, and dispersion of either $D=4 \mathrm{ps} / \mathrm{n}$ $\mathrm{m} / \mathrm{km}$ (non-zero dispersion shifted fiber (NZDSF)) or $D=17 \mathrm{ps} / \mathrm{nm} / \mathrm{km}$ (standard single-mode fiber $(\mathrm{SMF})$ ). Propagation in each fiber was obtained with the Manakov-based SSFA described above, and solved in absence of PMD with the desired nonlinear effect $\mathrm{ON}$. In the DM link we used a pre-compensating fiber of $-370 \mathrm{ps} / \mathrm{nm} @ D=4$ $\mathrm{ps} / \mathrm{nm} / \mathrm{km}$ and $-650 \mathrm{ps} / \mathrm{nm} @ D=17 \mathrm{ps} / \mathrm{nm} / \mathrm{km}$, in-line dispersion of $30 \mathrm{ps} / \mathrm{nm} / \mathrm{span}$, and a postcompensating fiber whose value set the overall dispersion $D_{T}=0$. In the noDM case only the post-compensating fiber was present to set $D_{T}=0$. All signals were generated with random SOPs and then wavelength multiplexed.

We studied each nonlinear effect individually by investigating for a homogeneous WDM PDMQPSK system with non-return to zero (NRZ) pulses both with DM and noDM: 1) the "bell curve" performance at 112Gbit/s (symbol rate $R=28$ Gbaud) with 19-channels and $50 \mathrm{GHz}$ spacing; 2) the nonlinear threshold (NLT) versus $R$ at fixed bandwidth efficiency $\eta=R / \Delta f$, i.e. at constant relative cross nonlinear effects ${ }^{5}$.
The standard DSP-based receiver included polarization recovery and phase estimation with the Viterbi algorithm $(\mathrm{V} \& \mathrm{~V})^{5}$, while we assumed zero frequency offset and zero laser phase noise.

Performance was measured in terms of the Qfactor of the central channel, extrapolated from the BER. BER was estimated from Monte Carlo simulations stopped after counting 100 errors. Bell curves were simulated using noise loading at the receiver, while nonlinear signal noise interaction (NSNI) was accounted only in the evaluation of the NLT, by distributing the optical noise on the line amplifiers.

\section{Gbit/s PDM-QPSK "bell curves"}

We compared the relative importance of XPM and XPolM using the proposed separation of effects in a 112 Gbit/s PDM-QPSK system, both with $\mathrm{DM}$ and noDM. In these simulations we used De Bruijn sequences of 1024 symbols for each of the 19 WDM channels, and applied noise loading, i.e., neglected NSNI. The number of V\&V taps was here $3+1+3$. The average $Q$-factor versus power is depicted in Fig. 1. Each curve is labeled with the specific active nonlinear effect. Label WDM represents the "true" case with all nonlinear effects ON, including FWM. We observe that XPoIM is the limiting factor in the DM case for both fiber types. This is in agreement with intuition, since in DM maps the NRZ-QPSK signal power 

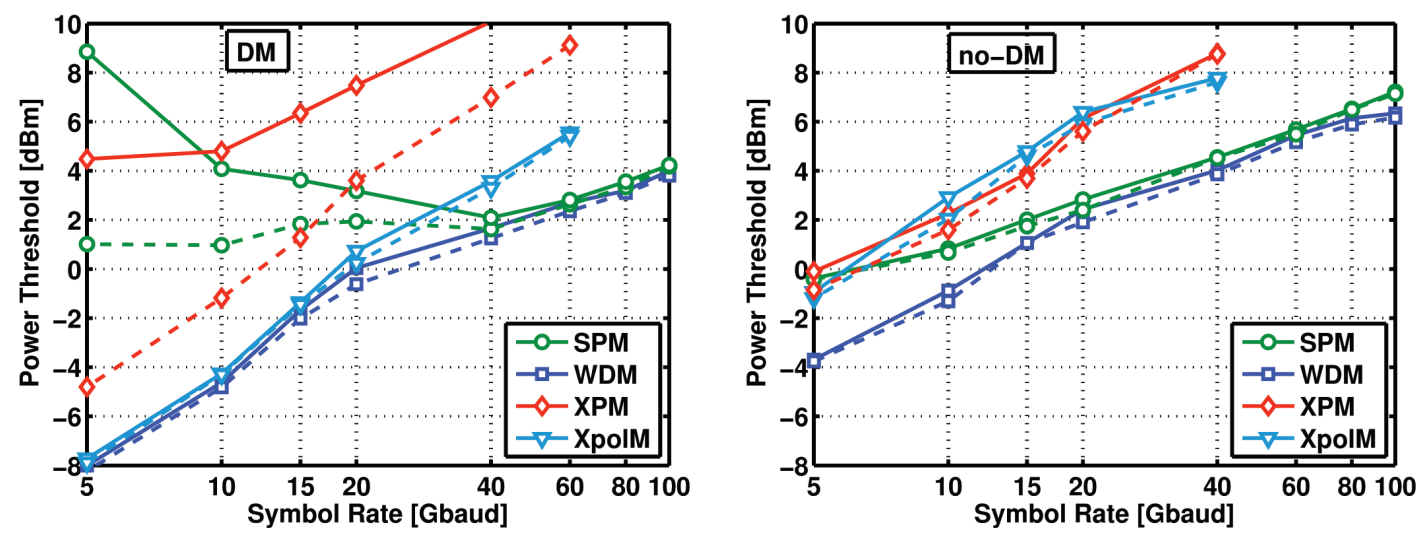

Fig. 2: NLT with each nonlinear effect acting individually in a 20x100 km SMF link with homogeneous WDM PDM-QPSK channels. Solid line: noise loading. Dashed lines: distributed noise (i.e., with NSNI).

experiences moderate fluctuations, while signal phase modulation makes the instantaneous SOP alternate among 4 different values, an aspect that is detrimental for XPolM. The noDM curves show a different behavior, with a lower gap between WDM and single channel because of a dominant SPM contribution. However, a gap between the single channel and the WDM case still exists, showing that cross-channel nonlinearities cannot be neglected. We note comparable contributions of XPM and XPolM, with opposite dominance in NZDSF and SMF. Note also that while the two DM cases are almost identical in absolute terms, with just more XPM in the NZDSF case, the noDM case indicates that SMF is a better choice.

\section{NLT versus baud rate}

We generalize here the previous investigations to a wider range of symbol rates $R$, by testing each nonlinear effect individually, including NSNI. We follow the same steps of ${ }^{5}$, to which we refer the reader for more details. Here the transmission fiber is SMF, and we used $13+1+13$ taps in $\mathrm{V} \& \mathrm{~V}$. The number of WDM channels was suitably scaled inversely ${ }^{5}$ with $R$. For a fair comparison among systems with different $R$, we express performance in terms of the NLT, i.e., the transmitted power at $1 \mathrm{~dB}$ of optical signal-to-noise ratio penalty at BER of $10^{-3}$. Fig. 2(left) shows NLT versus $R$ for the various nonlinear effects in the DM case. Solid lines are for noise loading, dashed lines for distributed noise (i.e., including NSNI). Label SPM indicates single channel $\mathrm{NLT}$, and we note that $\mathrm{NSNI}$ is significant up to 40 Gbaud. Regarding XPM, without NSNI the intensity is almost periodic and the XPM is thus well suppressed by the differential phase detection (XPM, solid); the intensity becomes aperiodic because of NSNI, inducing more penalty (XPM, dotted). However, XPoIM always dominates XPM. $\mathrm{XPolM}$ is insensitive to NSNI, since it mostly depends on the modulation-induced random orientation of the pivot ${ }^{3}$. XPoIM is clearly the dominant nonlinearity up to 20 Gbaud. At large $R$ SPM sets the performance, while between 20 and 40 Gbaud there is a trade-off between SPM and XPoIM.

Fig.2 (right) shows the NLT in a noDM link. We see two major differences from the DM case: 1) $\mathrm{NSNI}$ is always negligible; 2) XPM and XPoIM are comparable. However, the dominant impairment turns out to be SPM, even if cross-channel effects are not negligible at baudrates lower than 20 Gbaud, where they interact with SPM to set the overall NLT. Note that in noDM also the SPM NLT has a linearly increasing behavior, i.e., dispersion is efficiently used to mitigate nonlinearities; such a behavior appears even in DM at baudrates larger than 40 Gbaud with the same slope. We conclude that, whatever the link, increasing the baudrate is a good choice to improve the performance of DSP-based coherent PDM-QPSK.

\section{Conclusions}

We analyzed the individual impact of relevant nonlinear effects (SPM, XPM, XpoIM, NSNI) in PDM-QPSK links. We showed that XpolM dominates XPM in a wide range of setups, except in noDM links.

\section{References}

1 M. Karlsson et al., J. Lightw. Tech. 24, 4127 (2006).

2 J. Renaudier et al., Proc. ECOC'09, Tu.3.4.5 (2009).

3 M. Winter et al., J. Lightw. Tech. 27, 3739 (2009).

4 A. Bononi et al., Proc. ECOC'09, Th.10.4.6 (2009).

5 A. Bononi et al., Optical Fiber Tech. 16, 73 (2010).

6 C.R. Menyuk et al, J. Lightw. Tech. 24, 2806(2006).

7 P. Serena et al., "Optilux Toolbox", available at www.optilux.sourceforge.net 